\title{
General Medicine and Hospital Medicine: The Janus of Internal Medicine
}

\author{
Laurence F. McMahon Jr, MD, MPH' and Vineet Chopra, MD, MSC ${ }^{2}$ \\ 'Division of General Medicine, Department of Internal Medicine, University of Michigan, Ann Arbor, MI, USA; ${ }^{2}$ Division of Hospital Medicine, \\ Department of Internal Medicine, University of Michigan, Ann Arbor, MI, USA.
}

J Gen Intern Med 32(11):1168-9

DOI: $10.1007 / \mathrm{s} 11606-017-4182-\mathrm{z}$

(c) Society of General Internal Medicine 2017

I $\mathrm{n}$ this issue of JGIM, Miller and colleagues draw attention 1 to important trends in the evolution of academic general internal medicine. ${ }^{1}$ By examining the membership of the Society of General Internal Medicine (SGIM), they provide a snapshot not only of the Society, but also the trajectory of academic internal medicine. While one can belabor methodological concerns such as response rate and generalizability of the results, the overarching portrait of academic general medicine rings true: a growing proportion of SGIM members identify as hospitalists. Compared to non-hospitalists, Miller et al. found that hospitalists were younger, more junior in academic rank, and more often engaged in medical education roles. Additional noteworthy differences included the fact that hospitalists reported greater interest in developing clinical skills and less interest in advocacy than their non-hospitalist peers, and (somewhat surprisingly) they were more focused on research. Finally, hospitalist respondents were less likely to identify SGIM as their "professional home."

As the makeup of our membership changes, it is wise to pause and examine the unique perspective and contributions of academic general internists, regardless of their location of practice. Over a decade ago, Huddle et al. described academic departments of medicine as entities founded on the constructs of William Osler and the "consultant-generalist." The consultant-generalist was knowledgeable about anatomy, physiology, disease manifestations, and disease interactions. In time, the consultant-generalist evolved to become an expert integrative consultant - the academic generalist of today.

Over the course of the twentieth century, the field of internal medicine split into specialties and subspecialties emphasizing specific organs, sites of practice (e.g., emergency department), and patient age (e.g., geriatrics and adolescent medicine). With the establishment of the National Institutes of Health in 1948, a model focused largely on organ-based basic scientific exploration emerged. Academic departments of medicine in the

Published online September 25, 2017 1960s and '70s consequently focused on subspecialty groups - all of whom were housed within a unifying departmental structure. Paradoxically, training of internists shifted to being directed by subspecialists, even though many such specialty physicians functioned as consultant-generalists while attending on internal medicine wards. With renewed interest and funding in primary care by the federal government, divisions of general internal medicine were established in the 1970s. Additionally - by decree of the Residency Review Committee - continuity ambulatory experiences for internal medicine residents were also introduced. ${ }^{3,4}$ Thus, general internal medicine-staffed clinics became the model for patient care and for teaching residents the art of the consultantgeneralist in an ambulatory setting.

As subspecialists expanded their scientific base, they became less involved in managing patient conditions outside their area of specialization. Consequently, they also became less central to the training of internists in the consultantgeneralist tradition. The mantle of the Oslerian internist thus came to pass to general internal medicine faculty. Academic generalists not only embraced this role but also quickly became instrumental in fulfilling the educational, clinical, and quality missions of departments of medicine.

What are the key missions of a department of internal medicine today, and how should SGIM support academic generalists? The first and most obvious contribution of academic generalists is the education of students, residents, and fellows in wards and clinics. Today, the foundational training embodied within the Oslerian consultant-internist tradition falls largely to academic general internists. Importantly, the unique perspective that general internists adopt in evaluating a patient across organ boundaries is not only critical, but increasingly rare in academic departments of medicine. Additionally, both hospital medicine and primary care contextualize a patient's illness within their psychosocial and socioeconomic condition-while integrating information from multiple specialists into diagnostic and therapeutic decisions. In part, this skill set may help explain why junior hospital medicine faculty have gravitated to prominent educational roles, as noted in the survey.

Second, research has become a hallmark of academic internal medicine departments. Some departments of internal medicine view research as that which focuses on biomedical-based models of organ-based disease, as fostered by the National 
Institutes of Health (NIH). However, some departments have chosen to focus on studying health care, quality, and safety and delivery of health care - so-called health services research. Unlike organ-based models, this latter type of pragmatic research is often supported and sustained by internal funds from health systems. Additionally, this specific research focus may explain why so many hospitalist generalists report spending more of their academic effort on research. ${ }^{5}$

How can SGIM support and nurture members so that they meet their department's academic mission? A growing number of SGIM members now focus solely on practice and education in the hospital setting. At the same time, there has emerged a robust group of academic general internists practicing exclusively in the clinic setting. The fact that academic general internists in the Oslerian consultant-generalist model are thriving in SGIM-both in the clinic and in the hospital - should not be overlooked. Rather, it should be embraced. Unlike specialty organizations, SGIM should strive to accommodate the unique needs of all academic generalist faculty. From a practical perspective, this means looking across practice location boundaries for common threads in education, research, and clinical practice. While techniques in conducting clinically focused education at the bedside or at the exam table are different, the foundational approach to applying basic pathophysiology, integrating physical findings within the context of diagnostic tests, applying probabilistic approaches to differential diagnosis, and integrating psychosocial determinants are the same. Supporting those engaged in these activities - regardless of practice location - is thus SGIM's mission.

Similarly, creating a "home" for faculty to present the results of their research to peers for both appreciation and criticism should also represent a necessary part of SGIM's mission. As noted earlier, for academic generalists, a broad spectrum of research exists across the different departments of internal medicine. In practical terms, this means that research methods workshops, special research sessions, and topic-based research symposia must embrace not only nationally funded research, but also locally funded quality/safety and education research more typical of hospitalist investigators. Dedicated sessions that emphasize techniques such as LEAN, Six Sigma, and writing and disseminating quality improvement work would not only appeal to academic hospitalists, but it would be highly relevant to their professional development.

In conclusion, while the survey by Miller et al. highlights changes in the makeup of the membership of SGIM, it also serves as a wake-up call for our society. SGIM is in a singular position to be the natural home for academic general internists, whether their principal focus is education, research, or clinical care, or whether their site of practice is within or outside the hospital. As Wachter and Flanders noted 19 years ago, "Keeping both ambulatory and hospital-based academic generalists under one tent will require vision, commitment, and flexibility on the part of hospitalists, primary care physicians, and the leaders in our field." ${ }^{6}$ This must be the clarion call for SGIM.

Corresponding Author: Laurence F. McMahon, Jr, MD, MPH; Division of General Medicine, Department of Internal Medicine University of Michigan, Ann Arbor, MI, USA (e-mail: Imcmahon@umich.edu).

\section{Compliance with Ethical Standards:}

Conflict of Interest: Both authors declare that they have no conflict of interest.

\section{REFERENCES}

1. Miller CS, Fogerty RL, Gann J, et al. The growth of hospitalists and the future of the Society of General Internal Medicine: results from the 2014 membership survey. J Gen Intern Med. https://doi.org/10.1007/s11606017-4126-7.

2. Huddle TS, Centor R, Heudebert GR. American internal medicine in the 21st century: can an Oslerian generalism survive? J Gen Intern Med. 2003; 18:764-7.

3. Levinson W, Linzer $\mathbf{M}$. What is an academic general internist? Career options and training pathways. JAMA. 2002;288:2045-8.

4. Armstrong K, Keating NL, Landry M, Crotty BH, Phillips RS, Selker HP; for the Council of the Society of General Internal Medicine. Academic general internal medicine: a mission for the future. J Gen Intern Med. 2013:28:845-51.

5. Flanders SA, Saint S, McMahon LF, Howell JD. Where should hospitalists sit within the academic medical center? J Gen Intern Med. 2008;23:1269-72.

6. Wachter RM, Flanders S. The hospitalist movement and the future of academic general internal medicine. J Gen Intern Med. 1998;13:783-5. 